Tropical Journal of Pharmaceutical Research November 2018; 17 (11): 2189-2197

ISSN: 1596-5996 (print); 1596-9827 (electronic)

(1) Pharmacotherapy Group, Faculty of Pharmacy, University of Benin, Benin City, 300001 Nigeria.

Available online at http://www.tjpr.org

Original Research Article

http://dx.doi.org/10.4314/tjpr.v17i11.12

\title{
Cardioprotective effect of salvianolic acid B against isoproterenol-induced inflammation and histological changes in a cardiotoxicity rat model
}

\author{
Jun Liu, Liang Chen, Huihe Lu* \\ Department of Cardiovascular Medicine, The First Hospital of Nantong, Nantong 226000, China
}

*For correspondence: Email: TheresahThompsonja@yahoo.com; Tel: 0086-0513-85061272

Sent for review: 29 June 2018

Revised accepted: 26 August 2018

\begin{abstract}
Purpose: To investigate the cardioprotective role of salvianolic acid B (SAB-B) against isoproterenol (ISO)-induced cardiotoxicity in a rat model.

Methods: A total of 32 male rats were equally divided into four groups as follows: control group given saline only; rats treated with ISO (85 mg/kg; i.p.) for 2 consecutive days (MI model; ISO group); rats pretreated with $S A B-B(20 \mathrm{mg} / \mathrm{kg}$, i.p.) prior to exposure to ISO (SAB-B+ISO, treatment group), and rats administrated $S A B-B(20 \mathrm{mg} / \mathrm{kg}$, i.p.) for 28 days (SAB-B group).

Results: Pre-treatment with SAB-B for 28 days enhanced the levels of various antioxidants (CAT, SOD and GSH) and hemodynamic parameters, viz, systolic blood pressure (SBP), diastolic blood pressure $(D B P)$ and mean arterial pressure (MAP). Likewise, substantial decrease in the levels of lipid peroxidation product (MDA), inflammatory markers (NF-Kb, TNF- $\alpha, I L-1 \beta$ and IL-6), heart weight, heartto-body weight ratio, and serum cardiac markers (CPK and $L D H)$ were seen in SAB-B treated rats, relative to ISO-treated group. Rats in SAB-B group had significantly downregulated protein expressions of NF-Kb and TNF- $\alpha$, and significant reduction in histopathological changes (edema and necrosis) after 28 days of treatment.

Conclusion: SAB-B possesses potent cardio-protective potential against ISO-induced cardiotoxicity due to its antioxidant, anti-inflammatory and anti-necrotic properties.
\end{abstract}

Keywords: Salvianolic acid B, Isoproterenol, Antioxidants, Inflammatory markers, Cardioprotection

\begin{abstract}
This is an Open Access article that uses a funding model which does not charge readers or their institutions for access and distributed under the terms of the Creative Commons Attribution License (http://creativecommons.org/licenses/by/4.0) and the Budapest Open Access Initiative (http://www.budapestopenaccessinitiative.org/read), which permit unrestricted use, distribution, and reproduction in any medium, provided the original work is properly credited.

Tropical Journal of Pharmaceutical Research is indexed by Science Citation Index (SciSearch), Scopus, International Pharmaceutical Abstract, Chemical Abstracts, Embase, Index Copernicus, EBSCO, African Index Medicus, JournalSeek, Journal Citation Reports/Science Edition, Directory of Open Access Journals (DOAJ), African Journal Online, Bioline International, Open-J-Gate and Pharmacy Abstracts
\end{abstract}

\section{INTRODUCTION}

Cardiovascular disease (CVD) is one of the major causes of mortality and morbidity globally, and it accounts for $20 \%$ of all deaths. A report by World Health Organization (WHO) predicted that approximately 30 million people will die from CVD by the year $2030[1,2]$. Similarly, in China, the rate of CVD-related death has significantly increased as a result of modified lifestyle (sedentary pattern and western foods) and rapid urbanization [3]. Studies have shown that 
myocardial infarction (MI) is one of the pivotal reasons for all cardiovascular disease-related deaths $[4,5]$. Myocardial infarction results from prolonged blockage of blood supply via the coronary artery owing to ruptured atherosclerotic plaques and eventually results in oxidative stress and necrosis in myocytes $[6,7]$. The pathophysiology of $\mathrm{Ml}$ is still obscure. However, oxidative stress, inflammation, apoptosis, hypoxia and necrosis are crucial contributors to $\mathrm{MI}[8,9]$.

Isoproterenol (ISO) is a non-selective $\beta$ adrenergic agonist and synthetic catecholamine that imposes considerable oxidative stress on cardiomyocytes (after supramaximal dosage) due to auto-oxidation of catecholamine; it results in necrosis, edema and apoptosis, and ultimately in myocardial injury and myocardial infarction $[10,11]$. Studies have shown that the pathophysiologies of $\mathrm{MI}$ and ISO-induced $\mathrm{MI}$ are similar: both trigger various pathological events such as oxidative stress, inflammatory response, necrosis, apoptosis and mitochondrial dysfunction $[6,8]$. In the present study, an ISOinduced $\mathrm{Ml}$ model was used to assess the cardio-protective potential of salvianolic acid $B$ (SAB-B).

Salvianolic acid B (SAB-B) is a natural and abundant water-soluble phenolic acid found in the root/rhizome of Salvia militiorrhiza Bunge. Salvia militiorrhiza is a popular Chinese herb used in Traditional Chinese Medicine (TCM) for treating various ailments such as cerebrovascular and cardiovascular disease, and dysmenorrhea [12,13]. Salvianolic acid (SAB-B) exhibits a broad range of biological activities including anti-inflammatory, anti-apoptotic, antioxidant, anti-cancer and anti-diabetic, cardioprotective, neuroprotective, gastro-protective and renoprotective properties in various experimental models [14-16].

Previous studies showed that salvianolic acid-A, another phenolic acid from Salvia militiorrhiza exerted cardio-protective activity against isoproterenol-induced myocardial infarction in a rat model [17]. Furthermore, SAB-B exerts vascular protective action against experimentallyinduced acute myocardial infarction in a rat model $[18,19]$. However, no studies have carried out to elucidate the cardio-protective effect of salvianolic acid B against isoproterenol-induced myocardial infarction. Therefore, the present animal study was conducted to investigate the cardioprotective role of SAB-B) against isoproterenol (ISO) induced cardiotoxicity in rat model.

\section{EXPERIMENTAL}

\section{Chemicals}

Salvianolic acid B, isoproterenol hydrochloride, hematoxylin and eosin $(\mathrm{H} \& \mathrm{E})$ stain, pentobarbital sodium, and formalin were purchased from Sigma-Aldrich (MO, USA). Other chemicals and reagents used in the current study were of analytical grade.

\section{Experimental rats}

Thirty-two male, white Sprague-Dawley rats (mean weight $250 \pm 10 \mathrm{~g}$ ) were used in this study. The rats were housed in well-ventilated stage cage with unrestricted access to food and water and maintained at $23-24^{\circ} \mathrm{C}$, and humidity of $55-60 \%$ in an environment with $12 \mathrm{~h} / 12 \mathrm{~h}$ light/dark cycle. The experimental protocols and procedures used in this study were based on the guidelines reported by the National Institute of Health (NIH; MD, USA) [20], and were approved by the Animal Ethical Committee Board of The First Hospital of Nantong, China (approval no. FHN-16/026/A003).

\section{Preparation of MI model}

Isoproterenol hydrochloride (ISO) was injected i.p. at a dose of $85 \mathrm{mg} / \mathrm{kg}$ (dissolved in $0.89 \%$ saline solution) on the $29^{\text {th }}$ and $30^{\text {th }}$ day $(24 \mathrm{~h}$ interval) to induce MI.

\section{Experimental grouping}

Thirty-two male, white Sprague-Dawley rats were divided into 4 groups (8 rats/group). Rats administered only saline served as the control group, whereas rats treated with ISO $(85 \mathrm{mg} / \mathrm{kg}$, i.p.) for 2 consecutive days (MI model) served as ISO group. Rats pre-treated with SAB-B (20 $\mathrm{mg} / \mathrm{kg}$, i.p.) for 28 days prior to treatment with ISO served as SAB-B+ISO group (treatment group), whereas rats administered only SAB-B (20 $\mathrm{mg} / \mathrm{kg}$; i.p.) for 28 days comprised the SAB-B group.

\section{Sample collection}

All the rats were fasted overnight, and on the $31^{\text {st }}$ day the rats were weighed and anesthetized with pentobarbital sodium (50 mg/kg, i.p.). Blood was collected from the aorta in non-anticoagulant tubes and the rats were sacrificed by cervical decapitation. The myocardium was excised immediately from each rat, rinsed in ice-cold saline, dried and weighed. A portion of the cardiac tissue was homogenized in phosphate buffered saline (PBS; $7.4 \mathrm{pH}$ ). The homogenate 
was centrifuged at $10000 \times \mathrm{g}$ for $10 \mathrm{~min}$ at $4^{\circ} \mathrm{C}$, and the supernatant portion was used for biochemical analysis. The remaining portion of cardiac tissue was fixed in $10 \%$ formalin for assessment of any morphological changes. The blood samples collected were allowed to clot, and the serum samples were obtained by centrifuging at $3500 \times \mathrm{g}$ for $15 \mathrm{~min}$ at $4^{\circ} \mathrm{C}$. All the samples were stored at $-80^{\circ} \mathrm{C}$ prior to use.

\section{Biochemical analysis}

\section{Evaluation of hemodynamic parameters}

Twenty-four hours after the last administration of ISO $\left(31^{\text {st }}\right.$ day before sacrifice), the blood pressure of the rats was measured using NIBP200A (BIOPAC Systems, INC., CA, USA), a tail-cuff, non-invasive blood pressure monitor.

\section{Assay of cardiac antioxidants}

The activities of cardiac antioxidant enzymes glutathione peroxidase (GPx), catalase (CAT), and superoxide dismutase (SOD) were assayed using a commercial kit from Sangon Biotechnology (Shanghai, China), based on manufacturer's procedure.

\section{Determination of lipid peroxidation}

The levels of cardiac lipid peroxidation (LPO) product (malondialdehyde, MDA) was determined using assay kits from Nanjing Jiancheng Bioengineering Institute (Nanjing, China) based on manufacturer's protocol.

\section{Assessment of cardiac function}

The activities of serum lactate dehydrogenase $(\mathrm{LDH})$ and creatine kinase-MB (CK-MB) were measured with ELISA kit (Kangchen Biotechnology, Shanghai, China) in accordance with manufacturers' protocol. The levels of serum cardiac troponin $\mathrm{T}$ (cTn-T) were measured using Elecsys troponin-T Stat kit from Roche Diagnostics Ltd., (Risch-Rotkreuz, Switzerland) based on manufacturer's protocol.

\section{Measurement of cardiac inflammatory markers}

The concentration of cardiac inflammatory markers i.e., interleukins 1 (IL-1 $\beta$ ), tumour necrosis factor (TNF- $\alpha$ ), and interleukins 6 (IL-6) in cardiac tissue homogenate were measured using commercial ELISA kit from Neobioscience Technology, Co., Ltd (Beijing, China) based on supplier's instruction.

\section{Quantification of inflammatory markers by Immunoblot}

The supernatant from the cardiac tissue homogenate was used to extract the nuclear and cytosolic fractions using Nuclear and Cytoplasmic Extraction Kit (Guge Biotechnology; Wuhan, China). For the quantification of the protein expressions of TNF- $\alpha$ in cytosolic fraction and nuclear factor kappa B p65 subunit (NF-kB p65) in the nuclear fraction, RIPA buffer with 1 $\%$ protease inhibitor (Guge Biotechnology, Wuhan, China) was used for protein extraction, and protein was estimated using BCA protein kit (Bio-Rad Laboratories. Inc., CA, USA).

Thereafter, $40 \mu \mathrm{g}$ of protein (cardiac tissuecytosolic/nuclear fraction) were separated in 10 $\%$ SDS-PAGE and transferred onto polyvinylidene difluoride (PVDF) membrane. The membrane was blocked using Tris-phosphate buffered saline (TPBS) containing $5 \%$ skimmed milk and tween 20 . This was followed by probing the membrane overnight at $4{ }^{\circ} \mathrm{C}$ with primary antibodies i.e. rabbit monoclonal anti-NF-p65 (1:1500 dilution), anti-TNF- $\alpha$ (1:1200 dilution) and standard protein $\beta$-actin (1:1000 dilution) antibodies (Zhongshan Biotechnology, Beijing, China). Excess primary antibody was removed with TPBS, and finally the PVDF membrane was probed at $37^{\circ} \mathrm{C}$ for $1 \mathrm{~h}$ with secondary antimouse polyclonal horseradish peroxidase (HRP) antibody (1:10000 dilution) purchased from Cell Signaling Technology (Shanghai, China). The protein bands were visualized using enhanced chemiluminescent detection system (GE Healthcare Life Sciences; IL, USA) and quantified with Image $J$ software (ver 2.6) from $\mathrm{NIH}$ (MD, USA).

\section{Assessment of morphological changes}

Cardiac tissue fixed in formalin (10 \%) was dehydrated, fixed and embedded in liquid paraffin (wax) and made into a tissue block. The cardiac tissue block was cut into 3-5 $\mu \mathrm{m}$ diameter slices using microtome Cryo Leica EM (Leica Microsystem Inc., Buffalo, IL, USA), and bound to microscopic slides. Subsequently, the cardiac tissue slides were stained with $\mathrm{H}$ \& $\mathrm{E}$, and assessed for any morphological changes (edema, coagulated myonecrosis, and inflammatory cell infiltration) using a light microscope (Olympus Co., Tokyo, Japan) at $100 \times$ magnification.

\section{Statistical analysis}

Data are expressed as mean \pm standard error of mean (SEM, $n=8$ ). Differences between groups 
were analyzed with one way ANOVA, followed by Tukey's multi-comparison test. All statistical analyses were carried out using GraphPad Prism statistical package program software, version 6 (GraphPad Software, Inc., CA, USA). Values of $p$ $<0.05$ were considered statistically significant.

\section{RESULTS}

Table 1 shows the effect of SAB-B on heart weight, body weight and heart-to-body weight ratio in control and ISO-induced rats. The average values of heart weight and heart-to-body weight ratio were significantly higher $(p<0.01)$ in ISO-treated rats (Ml-model) than in the salinetreated control rats. However, the mean values of heart weight and heart-to-body weight ratio were significantly lower $(p<0.05)$ in the SAB-B group than in the ISO-exposed rats. Nevertheless, no appreciable changes were observed in body weights in any of the experimental rats.

The effect of SAB-B on hemodynamic parameters in control and ISO-induced rats are presented in Table 2. The levels of various hemodynamic parameters including diastolic arterial pressure (DAP), systolic arterial pressure (SAP) and mean arterial pressure (MAP) were markedly decreased by ISO-treatment $(p<0.01)$. However, treatment with SAB-B for 28 days significantly improved the levels of various hemodynamic parameters $(p<0.01)$.

Table 3 shows the effect of SAB-B on lipid peroxidation and cardiac antioxidants in control and ISO-treated rats. When compared with control rats, the ISO-treated rats had significant increase in MDA level $(p<0.01)$ and significant decreases in the activities of the endogenous antioxidants GPx, CAT, SOD $(p<0.01)$. However, pretreatment of the rats with SAB-B for 28 days led to significant reduction in levels of MDA $(p<0.05)$ and exponential increases in the activities of the various antioxidants, relative to the ISO-treated rats $(p<0.01)$.

Table 4 represents the effect of SAB-B on serum cardiac markers in control and ISO-treated rats. Significant increases $(p<0.01)$ in the levels of different serum cardiac diagnostic markers ( $\mathrm{LDH}$, CK-MB and cTnT) were seen in ISO-treated rats, when compared with control rats. In rats treated with SAB-B, followed by 2 days of exposure to ISO, there were significant decreases $(p<0.01)$ in the serum levels of different cardiac diagnostic markers.

Table 1: Effect of SAB-B on heart weight, body weight and heart-to-body weight ratio in control and ISO-treated experimental rats

\begin{tabular}{lcccc}
\hline Parameter & Control & ISO & SAB-B+ISO & SAB-B \\
\hline Heart weight $(\mathrm{g})$ & $0.625 \pm 0.09$ & $0.892 \pm 0.12^{\mathrm{a}^{\star x}}$ & $0.719 \pm 0.06^{\mathrm{b}^{\star}}$ & $0.630 \pm 0.08$ \\
Body weight $(\mathrm{g})$ & $254.67 \pm 10.50$ & $241.45 \pm 9.09^{\mathrm{a}}$ & $250.91 \pm 10.85^{\mathrm{b}}$ & $255.72 \pm 8.88$ \\
Heart-to-body weight & $0.245 \pm 0.02$ & $0.369 \pm 0.05^{\mathrm{a}^{\star n}}$ & $0.286 \pm 0.03^{\mathrm{b}^{\star}}$ & $0.246 \pm 0.03$
\end{tabular}

ratio (\%)

Values are expressed as mean \pm standard error of mean (SEM). " $p<0.05$; " $p<0.01$. 'a' represents the comparison between ISO-treated (MI model) and control rats, while 'b' represents comparison between SAB-B + ISO and ISO-treated rats

Table 2: Effect of SAB-B on hemodynamic parameters in control and ISO-induced experimental rats

\begin{tabular}{|c|c|c|c|c|}
\hline Parameter & Control & ISO & SAB-B + ISO & SAB-B \\
\hline DAP (mmHg) & $85.40 \pm 5.59$ & $55.75 \pm 4.12^{a^{a *}}$ & $73.35 \pm 5.87^{b}$ & $86.22 \pm 6.06$ \\
\hline $\mathrm{SAP}(\mathrm{mmHg})$ & $132.70 \pm 7.29$ & $84.79 \pm 10.08^{a^{\star \star}}$ & $118.67 \pm 10.94^{\mathrm{b}^{n \times}}$ & $131.55 \pm 8.50$ \\
\hline MAP $(\mathrm{mmHg})$ & $112.17 \pm 5.63$ & $69.65 \pm 5.93^{\mathrm{a}^{x \times}}$ & $96.29 \pm 6.05^{b}$ & $110.23 \pm 4.83$ \\
\hline
\end{tabular}

Values are expressed as mean \pm standard error of mean (SEM). ' $p<0.05$; " $p<01$. 'a' represents comparison between ISO-treated (MI model) and control rats, while 'b' represents comparison between SAB-B + ISO and ISO-treated rats

Table 3: Effect of SAB-B on lipid peroxidation product and cardiac antioxidants in control and ISO-treated experimental rats

\begin{tabular}{|c|c|c|c|c|}
\hline Parameter & Control & ISO & SAB-B+ISO & SAB-B \\
\hline MDA (nmol/mg protein) & $0.62 \pm 0.07$ & $0.93 \pm 0.10^{\mathrm{a}^{\star n}}$ & $0.78 \pm 0.09^{b^{n}}$ & $0.61 \pm 0.06$ \\
\hline CAT (U/mg protein) & $15.77 \pm 1.80$ & $9.21 \pm 1.15^{\mathrm{a}^{\mathrm{n}}}$ & $13.03 \pm 1.62^{\mathrm{D}^{n \times}}$ & $15.89 \pm 1.78$ \\
\hline SOD (U/mg protein) & $7.88 \pm 0.90$ & $4.26 \pm 0.62^{\mathrm{a}^{n \times}}$ & $6.33 \pm 0.80^{\mathrm{b}^{n \times}}$ & $8.02 \pm 0.95$ \\
\hline GPx ( $\mu \mathrm{g} / \mathrm{mg}$ protein) & $9.30 \pm 1.02$ & $6.74 \pm 0.89^{\mathrm{a}^{\star \pi}}$ & $8.06 \pm 0.97^{b^{\star \pi}}$ & $9.42 \pm 1.10$ \\
\hline
\end{tabular}

Values are expressed as mean \pm standard error of mean (SEM). ' $p<0.05$; " $p<0.01$. 'a' represents comparison between ISO-treated (MI model) and control rats, while 'b' represents comparison between SAB-B + ISO and ISO-treated rats 
Table 4: Effect of SAB-B on serum cardiac markers in control and ISO-induced experimental rats

\begin{tabular}{|c|c|c|c|c|}
\hline Parameter & Control & ISO & SAB-B+ISO & SAB-B \\
\hline cTn T (ng/mL) & $0.63 \pm 0.07$ & $1.94 \pm 0.18^{\mathrm{a}^{\star}}$ & $0.85 \pm 0.11^{\mathrm{b}}$ & $0.61 \pm 0.08$ \\
\hline CK-MB (IU/L) & $88.70 \pm 9.10$ & $172.02 \pm 19.25^{\mathrm{a}^{\mathrm{x}}}$ & $104.83 \pm 14.61^{\mathrm{b}^{\mathrm{xx}}}$ & $85.45 \pm 8.40$ \\
\hline LDH (IU/L) & $105.91 \pm 9.19$ & $196.85 \pm 17.34^{\mathrm{a}^{\star \pi}}$ & $131.59 \pm 15.07^{b^{\pi x}}$ & $108.44 \pm 13.11$ \\
\hline
\end{tabular}

Values are expressed as mean \pm standard error of mean (SEM). ' $p<0.05$; " $p<0.01$. 'a' represent the comparison between ISO-induced (MI model) and control rats, while 'b' represents comparison between SAB-B + ISO and ISO induced rats

Figure 1 shows the effect of SAB-B on inflammatory markers (cytokines) in control and ISO-induced rats. The concentrations of various inflammatory markers (IL-1 $\beta$, TNF- $\alpha$ and IL-6) were significantly increased $(p<0.01)$ in ISOinjected rats. However, administration of SAB-B for 4 weeks significantly abolished the increases in the ISO-induced increases in inflammatory markers, when compared with ISO-treated rats.

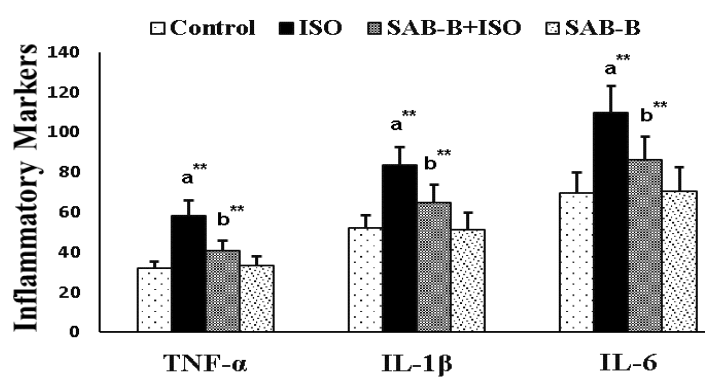

Figure 1: Effect of SAB-B on inflammatory markers (cytokines) in control and ISO-induced experimental rats. Values are expressed as mean \pm standard error of mean (SEM). $p<0.05$; $p<0.01$. 'a' represents comparison between ISO-treated (MI model) and control rats, while ' $b$ ' represents comparison between SAB-B + ISO and ISO-treated rats

The effect of SAB-B on protein expressions of TNF- $\alpha$ (cytosolic fraction) and NF-KB p65 (nuclear fraction) in cardiac tissue homogenate of control and ISO-treated rats are presented in Figure 2. The protein expressions of TNF- $\alpha$ and NF-KB p65 were significantly upregulated $(p<$ 0.01 ) in cardiac tissue homogenate of ISOtreated rats, relative to the control rats. However, rats treated with SAB-B (cardiac tissue homogenate) had significantly down-regulated ( $p$ $<0.01$ ) protein expressions of TNF- $\alpha$ and NF-KB $\mathrm{p} 65$, when compared to the ISO-treated rats.

Figure 3 depicts the effect of SAB-B on the histomorphology of heart tissue of control and ISOtreated experimental rats. Sections of heart tissue from the normal control rats displayed intact myofibres (Figure 3A). Disrupted myofibrils were evident in the cardiac slide of ISO-teated rats (Figure 3B), with edematous intracellular spaces (arrowed), coagulated necrosis and marked neutrophil granulocytes infiltration (circled). However, rats pre-treated with SAB-B (Figure 3C) showed better cardiac architecture with fewer number of disrupted myofibrils/necrosis and smaller edematous intracellular spaces (arrow mark). In contrast, the SAB-B-treated rats (Figure 3D) showed presence of normal cardiac architecture with prominent myofibril structure.

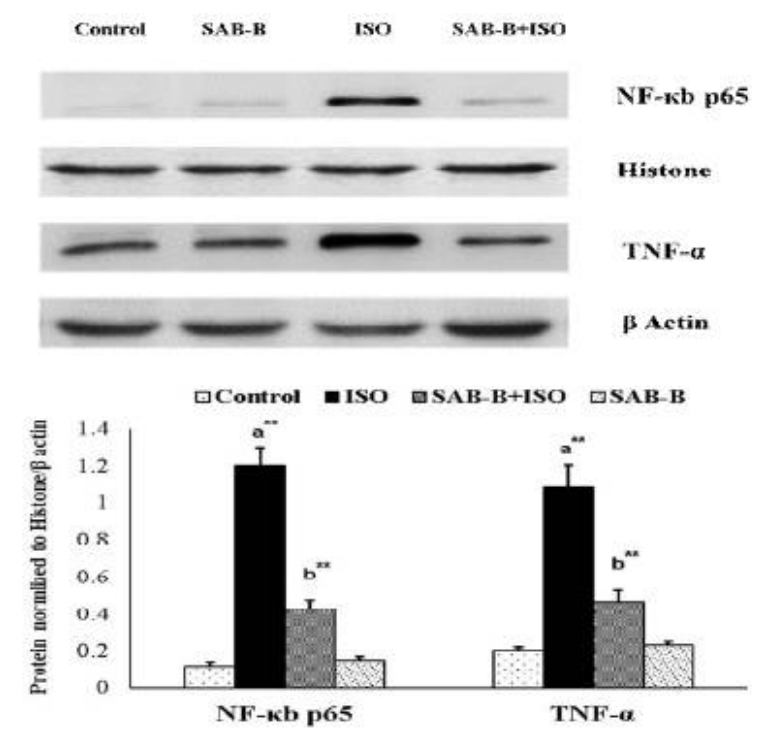

Figure 2: Effect of SAB-B on protein expressions of TNF- $\alpha$ and NF-KB p65 in cardiac tissue homogenate of control and ISO-treated experimental rats. Values are expressed as mean \pm standard error of mean (SEM). " $p<0.05 ;{ }^{* *} p<0.01$; 'a' represents comparison between ISO-treated (MI model) and control rats, while 'b' represents comparison between SAB-B + ISO and ISO-treated rats.

\section{DISCUSSION}

Till date, no studies have been carried out to elucidate the cardioprotective potential of salvianolic acid B against isoproterenol-induced $\mathrm{MI}$ in a rat model. The ISO-induced MI model was used in this study because it is the most reliable and the most accepted non-invasive method for inducing Ml. Furthermore, the pathophysiology of ISO-induced $\mathrm{MI}$ is almost similar to that of human MI [6]. The outcome of the present study showed that treatment with SAB-B significantly increased the activities of various antioxidants, improved hemodynamic parameters, and substantially decreased lipid peroxidation, inflammatory markers, heart weight, heart-to-body weight ratio, serum cardiac 
markers, and considerably reduced deleterious histopathological changes. Several studies have demonstrated that induction of ISO at supramaximal dose could trigger auto-oxidation of catecholamine, which leads to overproduction of free radicals (ROS) and lipid peroxidation in cardiomyocytes plasma membrane and ultimately necrosis and myocardial injury [21,22].
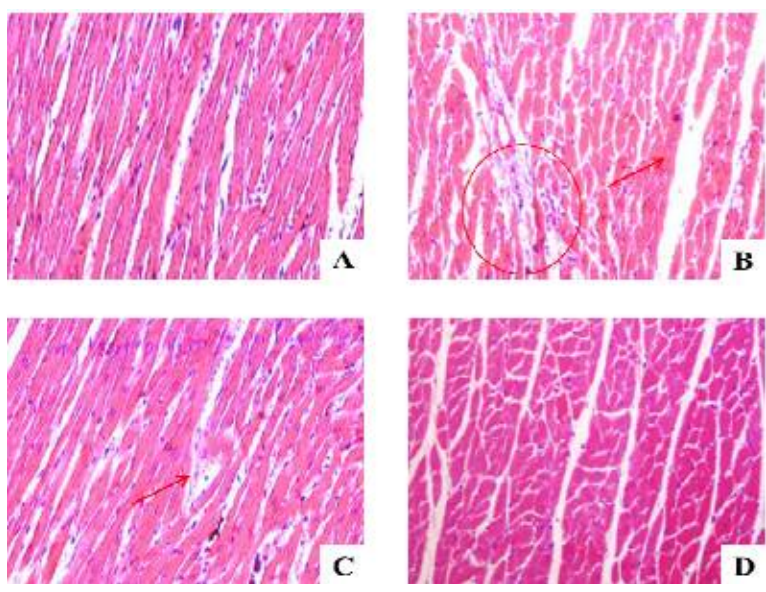

Figure 3: Effect of SAB-B on the morphology of heart tissue in control and ISO-treated rats. Sections of heart tissue from normal control rats $(A)$ displayed intact myofibres, while heart tissue section from the ISO-treated rats (B) portrayed high number of disrupted myofibrils, with edematous intracellular space (arrowed), coagulated necrosis and marked neutrophil granulocyte infiltration (circled). However, rats pre-treated with SAB-B (C) showed better cardiac architecture with fewer disrupted myofibrils/necrosis and smaller edematous intracellular spaces (arrowed). In contrast, rats treated with SAB-B alone had normal cardiac architecture (similar to control) with prominent myofibril structure (D)

The mean value of heart weight and heart-tobody weight ratio were significantly increased after ISO treatment due to oxidative stressinduced cardiac hypertrophy. This led to increased movement of water into cardiomyocytes and increased heart weight and heart-to-body weight ratio. On treatment with SAB-B, the oxidative stress due to ISO was markedly abolished, thereby abolishing the edematous condition or necrosis. Thus, the levels of heart weight and heart-to-body weight ratio were considerably reduced. Previous studies also indicated that treatment with salvianolic acid B and tanshinone IIA for 28 days significantly attenuated cardiac infarction hypertrophy (edema), thereby decreasing heart weight and heart-to-body weight ratio [23].

It has been well documented that isoproterenolinduced myocardial injury (due to oxidative stress) is contributed mostly by contractile dysfunction of the left ventricle (cardiac remodeling) which eventually leads to altered hemodynamic parameters especially blood pressure [24,25]. Thus, the levels of various hemodynamic parameters such as DAP, SAP, and MAP were markedly decreased in ISOtreated rats due to dysfunction in left ventricular contraction. It has been demonstrated that injection of ISO to rats considerably decreased arterial blood pressure (DAP, SAP, and MAP) [5]. Rats pre-treated with $S A B-B$ showed significant enhancement in the levels of various hemodynamic parameters, relative to ISOtreated rats. It has been reported that salvianolic acid B greatly improves cardiac output in rats induced with acute myocardial infraction (AMI). These results clearly suggest that SAB-B offers myocardial protection by abolishing ventricular dysfunction, thus preserving cardiac reflexes and cardiac output (blood pressure) due to its antioxidant and anti-inflammatory properties.

Oxidative stress (imbalance between oxidants and antioxidants) is the major contributor to MI. Hence, the level of lipid peroxidation product (MDA) was considerably increased in the ISOtreated rats. Increased levels of lipid peroxidation result in decreased levels of antioxidants like GPx, CAT, SOD. Administration of SAB-B for 28 days significantly reduced the levels of MDA owing to free radical scavenging activity/ antilipid peroxidation activity, restoring the antioxidants activity. Some previous studies have reported that the presence of two free hydroxyl groups in salvianolic acid $B$ is responsible for its free radical-quenching ability and its capacity for lowering lipid peroxidation and oxidative stress in the various models $[19,27,28]$.

The myocardium contains many cardiac marker enzymes and proteins such as creatine kinase (isoforms), lactate dehydrogenase and troponins which move out of the cardiomyocytes owing to increased lipid peroxidation. Thus, these enzymes/proteins are used for diagnosis of cardiac injury or damage [29]. The levels of serum CK-MB, LDH, and cTnT were markedly increased as a result of ISO exposure. As mentioned previously, ISO can elevate lipid peroxidation (oxidative stress) which results in rupture of cardiomyocytes and release of these cardiac markers from cardiomyocytes into the serum. Pre-treatment with SAB-B significantly decreased the levels of the serum cardiac markers owing to its free radical quenching ability. These results are consistent with the results of Lin and his colleagues [18], who reported that salvianolic acid $B$ decreases the serum levels of the cardiac markers LDH, CPK. These findings clearly indicate that SAB-B maintains membrane stability and thus inhibits 
the release or leakage of these cardiac markers into the blood.

Inflammatory response is one of the pivotal pathological factors that contribute to various cardiovascular diseases (CVDs) especially myocardial infarction. Studies have demonstrated that the expression of various proinflammatory markers (TNF- $\alpha$, IL- $1 \beta$ and IL-6) are directly associated with the activation of NF$\mathrm{KB}[30,31]$. During various inflammatory stimuli, inactive NF-KB is converted to active form which translocates to the nucleus and binds to promotor sequence, thus regulating the transcription of various pro-inflammatory cytokines i.e. TNF- $\alpha$, IL-1 $1 \beta$, IL-6 [32,33]. Therefore, different inflammatory markers (cytokines) are assayed by ELISA method, but the expressions of the active form of NF-KB p65 and TNF- $\alpha$ protein are quantified by western blot analysis.

The concentrations of various inflammatory markers such as IL-1 $\beta$, TNF- $\alpha$, IL- 6 were substantially increased in ISO-administered rats. Moreover, the protein expressions of NF-kB p65 and TNF- $\alpha$ (inflammatory markers) were markedly upregulated in ISO-administered rats, whereas rats treated with SAB-B for 28 days had significantly lowered the concentration of various inflammatory factors. These results are in agreement with the results of Wang and coworkers [34], who reported that salvianolic acid $B$ can significantly abolish increases in the inflammatory markers TNF- $\alpha, \quad$ IL-1 $\beta, \quad$ IL- 6 by downregulating the NF-KB signaling pathway. Likewise, Yang and his colleagues [35] reported that salvianolic acid $B$ inhibited the nuclear translocation of activated NF-kB p65 and thus downregulated the expression of various inflammatory cytokines (TNF- $\alpha$ ) in rabbit cardiac tissue.

Morphological analysis is conducted to assess if pathophysiological changes in experimental animals corroborate observed biochemical changes. Photomicrographs of heart tissue from the normal control rats showed intact myofibres, while those from ISO-tretaed rats showed disrupted myofibrils, with edematous intracellular space, coagulated necrosis and marked neutrophil granulocytes infiltration. However, rats pre-treated with SAB-B showed better cardiac architecture with fewer number of disrupted myofibrils/necrosis and small edematous intracellular space. This is agreement with the finding of $\mathrm{Xu}$ et al [26] who found that salvianolic acid $B$ intervention attenuated myocardial injury in $\mathrm{AMI}$ rat model.

\section{Study limitations}

The present study has some limitations such as non-investigation of myocardial infarct size, lipid profile and mitochondrial dysfunction.

\section{CONCLUSION}

The results obtained in the present animal study indicate that salvianolic acid B enhances the levels of antioxidants, and improves hemodynamic parameters in a rat model of MI. In additin, it mitigates $\mathrm{Ml}$-induced lipid peroxidation, downregulates inflammatory markers, decreases serum cardiac markers, and reduces histopathological changes in rats. However, further studies are required to validate these findings.

\section{DECLARATIONS}

\section{Acknowledgement}

The authors would like to thank the financial committee members of The First Hospital of Nantong for aiding this study.

\section{Conflict of interest}

No conflict of interest is associated with this study

\section{Authors' contribution}

This study was conducted by all the three authors (Jun Liu, Liang Chen and Huihe Lu) named in this manuscript and all liabilities pertaining to claims relating tothe contents of this manuscript will be borne by the authors. Jun Liu, Liang Chen contributed equally. Jun Liu and Huihe Lu concepted/designed this study. Liang Chen and Huihe Lu conducted this animal experiment. Jun Liu and Liang Chen involved in data collection as well as carried out statistical analysis. Liang Chen and Jun Liu both drafted/edited this manuscript.

\section{REFERENCES}

1. Ziaee $M$, Khorrami $A$, Ebrahimi $M$, Nourafcan $H$, Amiraslanzadeh M, Rameshrad M, Garjani M, Garjani A. Cardioprotective effects of essential oil of Lavandula angustifolia on isoproterenol-induced acute myocardial infarction in rat. Iran J Pharm Res 2015; 14(1): 279-289.

2. Evran B, Karpuzoğlu $H$, Develi S, Kalaz EB, SolukTekkeşin M, Olgaç V, Doğru-Abbasoğlu S, Uysal M. Effects of carnosine on prooxidant-antioxidant status in heart tissue, plasma and erythrocytes of rats with 
isoproterenol-induced myocardial infarction. Pharmacol Rep 2014; 66(1): 81-86.

3. Wei-Wei CH, Run-Lin GA, Li-Sheng LI, Man-Lu ZH, Wen WA, Yong-Jun WA, Zhao-Su WU, Hui-Jun LI, DongFeng GU, Yue-Jin YA, Zhe Zheng LX. China cardiovascular diseases report 2015: a summary. J Geriatr Cardiol 2017; 14(1): 1-10.

4. Lopez AD, Murray CC. The global burden of disease, 1990-2020. Nat Med 1998; 4(11): 1241-1243.

5. Mnafgui $K$, Hajji R, Derbali F, Gammoudi A, Khabbabi G, Ellefi $H$, Allouche $N$, Kadri A, Gharsallah N. Antiinflammatory, antithrombotic and cardiac remodeling preventive effects of Eugenol in Isoproterenol-induced myocardial infarction in Wistar rat. Cardiovasc Toxicol 2016; 16(4): 336-344.

6. Othman Al, Elkomy MM, El-Missiry MA, Dardor M. Epigallocatechin-3-gallate prevents cardiac apoptosis by modulating the intrinsic apoptotic pathway in isoproterenol-induced myocardial infarction. Eur $J$ Pharmacol 2017; 794: 27-36.

7. Kareem MA, Krushna GS, Hussain SA, Devi KL. Effect of aqueous extract of nutmeg on hyperglycaemia, hyperlipidaemia and cardiac histology associated with isoproterenol-induced myocardial infarction in rats. Trop $J$ Pharm Res 2009; 8(4): 337-344.

8. Goyal SN, Sharma C, Mahajan UB, Patil CR, Agrawal YO, Kumari S, Arya DS, Ojha S. Protective effects of cardamom in isoproterenol-induced myocardial infarction in rats. Int $\mathrm{J} \mathrm{Mol} \mathrm{Sci} \mathrm{2015;} \mathrm{16(11):} \mathrm{27457-}$ 27469.

9. Burke AP, Virmani R. Pathophysiology of acute myocardial infarction. Med Clin 2007; 91(4): 553-572.

10. Widyaningsi W, Pramono S, Widyarini S, Sugiyanto $D$. Cardioprotective effect of melatonin-standardized ethanol extract of Ulva lactuca $L$ against acute myocardial infarction in rats induced by isoproterenol. Trop J Pharm Res 2016; 15(8): 1723-1729.

11. Liu YT, Jia HM, Chang X, Ding G, Zhang HW, Zou ZM. The metabolic disturbances of isoproterenol induced myocardial infarction in rats based on a tissue targeted metabonomics. Mol BioSystems 2013; 9(11): 28232834.

12. Cao W, Guo XW, Zheng HZ, Li DP, Jia GB, Wang J. Current progress of research on pharmacologic actions of salvianolic acid B. Chin J Integr Med 2012; 18(4): 316-20.

13. Xun C, Wang S, Chen G, Hu Y, Xie J, Lv D. Salvianolic Acid B Ameliorates Motor Dysfunction in Spinal Cord Injury Rats. Trop J Pharm Res 2014; 13(1): 53-59.

14. Wang $J$, Xiong $X$, Feng $B$. Cardiovascular effects of salvianolic acid B. Evid Based Complement Alternat Med 2013; 2013.

15. Ma ZG, Xia HQ, Cui SL, Yu J. Attenuation of renal ischemic reperfusion injury by salvianolic acid $B$ via suppressing oxidative stress and inflammation through PI3K/Akt signaling pathway. Braz J Med Biol Res 2017; 50(6); e5954.
16. Chen J, Zhou W, Zhou Z, Yuan T, Li B, Zheng Y. Protective effect of salvianolic acid $B$ against intestinal ischemia reperfusion-induced injury in a rat model. Trop J Pharm Res 2017; 16(10): 2431-2438.

17. Wang SB, Tian S, Yang F, Yang HG, Yang $X Y, D u G H$. Cardioprotective effect of salvianolic acid $A$ on isoproterenol-induced myocardial infarction in rats. Eur $\mathrm{J}$ Pharmacol 2009; 615(1-3): 125-132.

18. Lin C, Liu Z, Lu Y, Yao Y, Zhang Y, Ma Z, Kuai M, Sun X, Sun $S$, Jing $Y, Y u$ L. Cardioprotective effect of Salvianolic acid $B$ on acute myocardial infarction by promoting autophagy and neovascularization and inhibiting apoptosis. J Pharm Pharmacol 2016; 68(7): 941-952.

19. Xue L, Wu Z, Ji XP, Gao XQ, Guo YH. Effect and mechanism of salvianolic acid $B$ on the myocardial ischemia-reperfusion injury in rats. Asian Pac J Trop Med 2014; 7(4): 280-4.

20. National Research Council. Guide for the care and use of laboratory animals. National Academies Press; Washington 2010: pp1-209.

21. Li H, Song F, Duan LR, Sheng JJ, Xie YH, Yang Q, Chen $Y$, Dong $Q Q$, Zhang BL, Wang SW. Paeonol and danshensu combination attenuates apoptosis in myocardial infarcted rats by inhibiting oxidative stress: Roles of Nrf2/HO-1 and PI3K/Akt pathway. Sci Rep 2016; 6: 23693.

22. Meeran MF, Jagadeesh GS, Selvaraj P. Thymol attenuates inflammation in isoproterenol induced myocardial infarcted rats by inhibiting the release of lysosomal enzymes and downregulating the expressions of proinflammatory cytokines. Eur J Pharmacol 2015; 754: 153-161.

23. Pan $C$, Lou $L$, Huo $Y$, Singh $G$, Chen $M$, Zhang $D$, Wu A, Zhao $M$, Wang $S$, Li J. Salvianolic acid $B$ and tanshinone IIA attenuate myocardial ischemia injury in mice by NO production through multiple pathways. Ther Adv Cardiovasc Dis 2011; 5(2): 99-111.

24. Zhou $R, X u Q$, Zheng $P$, Yan L, Zheng J, Dai G. Cardioprotective effect of fluvastatin on isoproterenolinduced myocardial infarction in rat. Eur $J$ Pharmacol 2008; 586(1-3): 244-250.

25. Mohanty I, Arya DS, Dinda A, Talwar KK, Joshi S, Gupta SK. Mechanisms of cardioprotective effect of Withania somnifera in experimentally induced myocardial infarction. Basic Clin Pharmacol Toxicol 2004; 94(4): 184-190.

26. Xu L, Deng Y, Feng L, Li D, Chen X, Ma C, Liu X, Yin J, Yang $M$, Teng F, Wu W. Cardio-protection of salvianolic acid $B$ through inhibition of apoptosis network. PloS one 2011; 6(9): e24036.

27. Jiang YF, Liu ZQ, Cui W, Zhang WT, Gong JP, Wang $X M$, Zhang $Y$, Yang MJ. Antioxidant effect of salvianolic acid $B$ on hippocampal CA1 neurons in mice with cerebral ischemia and reperfusion injury. Chin $\mathrm{J}$ Integr Med 2015; 21(7): 516-522.

28. Pan CS, Liu YH, Liu $Y Y$, Zhang $Y$, He $K$, Yang $X Y$, Hu $B H$, Chang $X$, Wang $M X$, Wei $X H$, Fan JY. Salvianolic

Trop J Pharm Res, November 2018; 17(11): 2196 
acid $B$ ameliorates lipopolysaccharide-induced albumin leakage from rat mesenteric venules through Srcregulated transcellular pathway and paracellular pathway. PloS one 2015; 10(5): e0126640.

29. Li H, Xie YH, Yang $Q$, Wang SW, Zhang BL, Wang JB, Cao W, Bi LL, Sun JY, Miao S, Hu J. Cardioprotective effect of paeonol and danshensu combination on isoproterenol-induced myocardial injury in rats. Plos one 2012; 7(11): e48872.

30. Lawrence $T$. The nuclear factor NF-KB pathway in inflammation. Cold Spring Harb Perspect Biol 2009; 1(6): a001651.

31. Cho SY, Park SJ, Kwon MJ, Jeong TS, Bok SH, Choi WY, Jeong WI, Ryu SY, Do SH, Lee CS, Song JC. Quercetin suppresses proinflammatory cytokines production through MAP kinases and NF-KB pathway in lipopolysaccharide-stimulated macrophage. Mol Cell Biochem 2003; 243(1-2): 153-160.
32. Carlson CG, Dole E, Stefanski C, Bayless D. The effect of specific IKK $\beta$ inhibitors on the cytosolic expression of IKB- $\alpha$ and the nuclear expression of $p 65$ in dystrophic (MDX) muscle. Am J Trans Res 2014; 7: 670-682.

33. Hoffmann A, Natoli $G$, Ghosh G. Transcriptional regulation via the NF-KB signaling module. Oncogene 2006; 25(51): 6706.

34. Wang $Y$, Chen G, Yu X, Li Y, Zhang L, He Z, Zhang N, Yang $X$, Zhao $Y$, Li N, Qiu $H$. Salvianolic acid $B$ ameliorates cerebral ischemia/reperfusion injury through inhibiting TLR4/MyD88 signaling pathway. Inflammation 2016; 39(4): 1503-1513.

35. Yang F, Chen Z, Lian Z, Guo Y, Liu G, Dong G. Inhibitory effects of salvianolic acid $B$ on myocardial cellular nuclear transfer of NF-KB p65 and the expression of TNF- $\alpha$ during ischemia-reperfusion in rabbits hearts in vivo [J]. China J Modern Med 2009; 5: 10-16. 